\title{
Molecular field calculation of the magnetic structure in erbium
}

\author{
J Jensen \\ Department of Theoretical Physics, 12 Parks Road, Oxford OX1 3PQ and AEK Research \\ Establishment, Risø, Roskilde, Denmark
}

Received 17 October 1975

\begin{abstract}
A molecular-field calculation of the magnetic configurations in $\mathrm{Er}$ is found to reproduce the neutron diffraction results of the three different magnetic phases and to give a reasonable fit to the magnetization data at $4.2 \mathrm{~K}$. The two-ion coupling is considered to be described by the inter-planar coupling parameters deduced from the dispersion of the spin waves in the low temperature conical phase. The four (effective) crystal-field parameters are determined by the fit to the experimental data to be (in units of $\mathrm{meV} /$ ion):

$$
\begin{aligned}
& B_{20}=-1.75 \times 10^{-2} \quad B_{40}=-4.75 \times 10^{-5} \\
& B_{60}=1.06 \times 10^{-6} \quad B_{66}=-6.9 \times 10^{-6} .
\end{aligned}
$$

Projecting the magnetic moments present in the intermediate phase of $\operatorname{Er}(18-52.4 \mathrm{~K})$ to a common origin the calculations indicate that the hodograph of the moments is very close to an ellipse that has its major axis parailel to the $c$-axis.
\end{abstract}

\section{Introduction}

Neutron diffraction studies of the magnetic structure of $\operatorname{Er}$ (Cable et al 1965, Habenschuss et al 1974, Atoji 1974) have revealed the existence of three distinct regions of long-range order.

(i) For $84 \cdot 4 \mathrm{~K}>T>52 \cdot 4 \mathrm{~K}$.

The $c$ axis moments are ordered in a sinusoidal structure with the wavevector parallel to the $c$ axis and a period of approximately seven atomic layers. As the temperature is decreased higher-order odd harmonics are gradually developed.

(ii) For $52 \cdot 4 \mathrm{~K}>T>18 \mathrm{~K}$.

A modulated ordering of the basal plane components (with a wavevector equal to that of the $c$ axis component) is superimposed on the $c$ axis sinusoidal structure.

(iii) For $T<18 \mathrm{~K}$.

A $c$ axis cone structure of a period close to eight atomic layers.

The neutron diffraction measurements define unambiguously the magnetic structures occurring in the high and the low temperature phases, (i) and (iii). The appearance of the higher-order odd harmonics in the $c$ axis modulated phase shows that the 
$c$ axis component of the moments approach a square wave. The square wave represents a compromise between the two-ion coupling, which favours a modulated structure with one fundamental wavevector, and the axial anisotropy favouring a $c$ axis alignment of the moments. The gradual development of the higher harmonics, as observed in Er, is predicted by the molecular field (MF) calculations of Miwa and Yosida (1961) and of Nagamiya (1967) who found that close to $T_{\mathrm{N}}$ (the Néel temperature for the $c$ axis component) the $(2 v+1)$-harmonic should be proportional to $\left(T_{N}-T\right)^{v+1}, v=0,1, \ldots$

The intermediate phase between the Néel temperature of the basal plane component, $T_{N_{1}}$, and the Curie temperature, $T_{c}$, is not fully characterized by the neutron experiments. Based on their MF calculations Miwa and Yosida (1961) proposed that only one of the components in the basal plane should deviate appreciably from zero. This configuration corresponds to a hodograph of the moments which is an ellipse that has its major axis along the $c$ direction. Cable et al (1965) argue that this configuration is not likely to occur because it seems to favour one direction in the basal plane in spite of the unimportance of the hexagonal anisotropy within this temperature range. They consider instead a model for which the major axis is tilted away from the $c$ axis so that both basal-plane components oscillate to execute a regular spiral. Both structures may be considered as modified versions of the tilted helix, the model which has been elaborated by Sherrington (1973). One of the aims of the present paper is to elucidate this question in more detail.

For this purpose we have attempted to establish a realistic MF model for $\mathrm{Er}$ with a use of a minimal number of variable parameters. A realistic model demands the use of a two-ion Hamiltonian which is in accordance with the dispersion relation of the spin waves propagating along the $c$ direction in the conical phase, as measured by Nicklow et al (1971). The apparent importance of two-ion anisotropy in Er (Nicklow et al 1971. Jensen 1974) introduces some arbitrariness in the interpretation of the spin wave data. However, we use the two-ion inter-planar coupling coefficients deduced by Jensen (1974) as representing the most adequate ones. The specification of the two-ion coupling and a neglect of magnetoelastic interactions leave four crystalfield and one intra-planar two-ion parameters as variables. These five parameters are determined by characteristic features of the magnetization data at $4.2 \mathrm{~K}$ and by the values of the two Néel temperatures. A determination of the parameters in this way results in a MF Hamiltonian which reproduces reasonably well most of the experimental data endowing the calculated structure of the intermediate phase with a certain amount of credibility.

\section{Molecular-field Hamiltonian}

The ground state value of the magnetic moments in Er at low temperatures (below $T_{c}$ ) is close to their saturation value. The dispersion relation of the spin waves propagating parallel with the $c$ axis in the cone phase of Er has been measured by Nicklow et al (1971) at $4.5 \mathrm{~K}$. The $q$ dependence of the spin wave energies exhibits a strong influence of two-ion anisotropy which is accounted for by the following two-ion Hamiltonian (Jensen 1974):

$$
\mathscr{H}_{11}=-\frac{1}{2} \sum_{i \neq j} \mathscr{f}(i j) J_{i} \cdot J_{j}-\frac{1}{2} \sum_{i \neq j} \mathscr{K}_{22}(i j) \frac{1}{8 J_{1}^{2}}\left[\left(J_{i}^{+} J_{j}^{-}\right)^{2}+\left(J_{i}^{-} J_{j}^{+}\right)^{2}\right]
$$


Table 1. The planar coupling coefficients of $\mathscr{f}(q) . \mathscr{J}^{\prime}(q)$, and $\mathscr{K}_{22}(q)$ (all values are in $\mathrm{meV})$.

\begin{tabular}{llrl}
\hline$m$ & \multicolumn{1}{c}{$\mathscr{I}_{m}$} & \multicolumn{1}{c}{$\mathscr{f}_{m}^{\prime}$} & \multicolumn{1}{c}{$\mathscr{K}_{m}$} \\
\hline 0 & 0.1716 & 0.1792 & 0 \\
1 & 0.0601 & 0.0587 & -0.0282 \\
2 & -0.0176 & -0.0223 & -0.0272 \\
3 & -0.0024 & -0.0048 & -0.0317 \\
4 & -0.0111 & -0.0130 & 0.0136 \\
5 & -0.0108 & -0.0080 & -0.0411 \\
6 & -0.0025 & 0.0013 & - \\
\hline
\end{tabular}

where $J_{i}$ is the total angular moment of the $i$ th ion. $J^{ \pm}$are defined with respect to a coordinate system with the $z$ axis along the $c$ direction and

$$
J_{n}=\left(J-\frac{1}{2}\right)(J-1) \ldots\left(J-\frac{1}{2} n\right) .
$$

The inter-planar coupling coefficients, $\mathscr{F}_{m}$, defined by

$$
\mathscr{J}(0)-\mathscr{J}(q)=2 \sum_{m>0} \mathscr{I}_{m}[1-\cos (m q c / 2)]
$$

and similar for $\mathscr{K}_{m}$, $(c$ is the lattice parameter) are given in table 1 and are those determined by Jensen (1974) from the experimental results of Nicklow et al (1971). The actual $q$ dependence of the two coupling parameters are displayed in figure 1 .

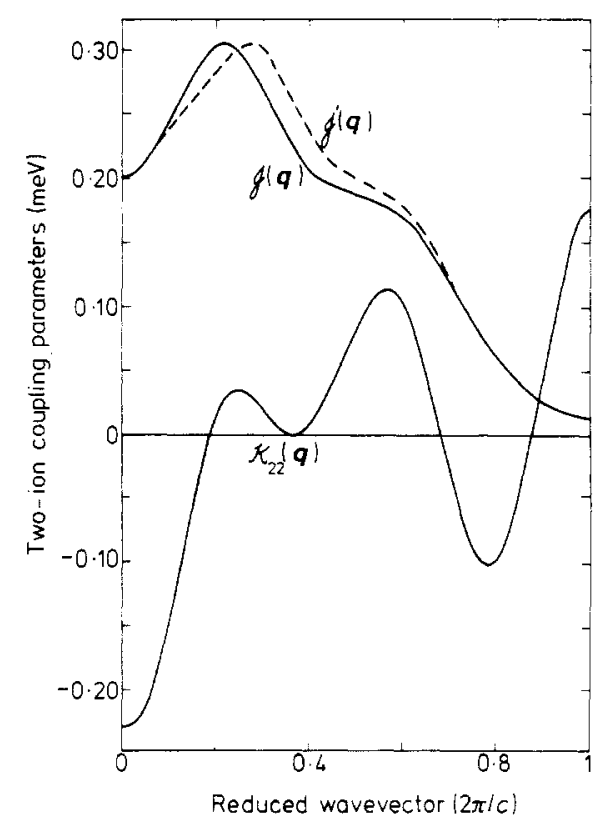

Figure 1. The $q$ dependence of the two-ion coupling parameters in the $c$ direction of Er (Jensen 1974). $\mathscr{J}(q)$ and $\mathscr{J}^{\prime}(q)$ are the isotropic couplings used in the cases of a structure with a period of eight and seven hexagonal layers respectively, $\mathscr{K}_{22}(q)$ is the anisotropic component. 
The wavevector, $Q$, of the conical structure is determined by the maximum value of the $q$ dependent part of the interaction,

$$
j(\boldsymbol{q})=\mathscr{J}(\boldsymbol{q})+\frac{1}{4} \mathscr{K}_{22}(2 \boldsymbol{q}) \sin ^{2} \theta
$$

where $\theta$ is the cone angle. The length of the wavevector in $\mathrm{Er}$ is observed to be $0 \cdot 24(2 \pi / c)$ which corresponds very closely to a period of eight atomic layers. A specific MF calculation of the magnetic structure requires a determination of the moments of each different layer in the period (corresponding to a variation of the molecular fields). A simplification of this problem is achieved by assuming a commensurate structure. Because of the length of the period ( $7-8$ hexagonal layers) this assumption is thought to be unimportant except for the introduction of a possible phase dependence of the (non-regular) configuration (see later, the response of such a system would be an average with respect to this phase dependence). The cone configuration of $\mathrm{Er}$ is assumed to be invariant to a displacement by eight atomic layers. In the high temperature phase the fundamental wavevector is observed to be very close to $0 \cdot 286(2 \pi / c)$ (Cable et al 1965, Habenschuss et al 1974, Atoji 1974) corresponding to a seven layer structure. Although an eight layer structure may still be considered as a fair approximation we have assumed a structure with a period of seven layers in both the high temperature and the intermediate phase (above $T_{c}$ ) because this allows a separation of the third and fifth harmonics, which is not possible in the case of an eight layer structure. The stabilization of the seven layer structure requires that $j(q)$ is at its maximum at $q=(4 \pi / 7 c)$. To accomplish this without interfering too much with the spin wave parameters we replace $\mathscr{F}(\boldsymbol{q})$ by $\mathscr{J}^{\prime}(\boldsymbol{q})$ in the case of a seven layer structure (above $\left.T_{c}\right) . \mathscr{J}^{\prime}(q)$ is obtained simply by displacing the maximum of $\mathscr{J}(\boldsymbol{q})$ to the position wanted. $\mathscr{J}^{\prime}(\boldsymbol{q})$ is shown by the broken line in figure 1 and the corresponding interplanar coefficients are given in table 1.

The two-ion Hamiltonian, (1), is transformed to an effective single-ion Hamiltonian by the MF approximation. The thermal expectation value of the $\alpha$ th component of the $i$ th moment is denoted $\left\langle J_{i}^{x}\right\rangle$ and the molecular fields are given by

$$
\begin{aligned}
& \mathscr{J}_{i}^{\alpha}=\sum_{j} \mathscr{J}(i j)\left\langle J_{j}^{\alpha}\right\rangle=\sum_{m=-6}^{+6} \mathscr{J}_{|m|}\left\langle J_{i+m}^{\alpha}\right\rangle \\
& \mathscr{K}_{i}^{ \pm}=\frac{1}{8 J_{1}^{2}} \sum_{m=-5}^{+5} \mathscr{K}_{|m|}\left\langle\left(J_{i+m}^{ \pm}\right)^{2}\right\rangle
\end{aligned}
$$

where the translation symmetry within a certain hexagonal layer is utilized $(i+m$ means an ion sited on the $(i+m)$ th layer). The MF Hamiltonian for the $i$ th ion then reads

$$
\begin{aligned}
\mathscr{H}_{\mathrm{II}}^{i}=-\sum_{x} \mathscr{J}_{i}^{\alpha} J_{i}^{\alpha}-\mathscr{K}_{i}^{+}\left(J_{i}^{-}\right)^{2}-\mathscr{K}_{i}^{-}\left(J_{i}^{+}\right)^{2}+\frac{1}{2}\left[\sum_{x} \mathscr{J}_{i}^{\alpha}\left\langle J_{i}^{\alpha}\right\rangle+\mathscr{K}_{i}^{+}\left\langle\left(J_{i}^{-}\right)^{2}\right\rangle\right. \\
\left.+\mathscr{K}_{i}^{-}\left\langle\left(J_{i}^{+}\right)^{2}\right\rangle\right] .
\end{aligned}
$$

The determination of the molecular fields, (3), requires a knowledge of the intra-planar coupling coefficients $\mathscr{F}_{0}$ and $\mathscr{K}_{0}$ or equivalently

$$
\mathscr{J}(0)=\sum_{m=-6}^{+6} \mathscr{J}_{|m|}
$$

and $\mathscr{K}_{22}(0) . \mathscr{I}_{0}$ is considered to be a variable parameter, whereas the effect of $\mathscr{K}_{0}$ is very similar to that of the crystal-field parameter $B_{40}$ except for a slightly different 
temperature dependence. In these calculations we have no possibility of isolating the pure crystal-field parameters from the effects of anisotropic two-ion couplings, we have, therefore, somewhat arbitrarily, assumed that $\mathscr{K}_{0}$ is zero (as also used in figure 1).

The possible contributions of two-ion anisotropic couplings and the neglect of magnetoelastic effects imply that the crystal-field parameters defined by the following single-ion Hamiltonian

$$
\mathscr{H}_{\mathrm{I}}^{i}=B_{20} \mathrm{O}_{2}^{0}+B_{40} \mathrm{O}_{4}^{0}+B_{60} \mathrm{O}_{6}^{0}+B_{66} \mathrm{O}_{6}^{6}
$$

have to be considered as effective ones. The operators, $\mathrm{O}_{l}^{m}$, appearing in (5) are Stevens operators (see e.g. Lindgard and Danielsen 1974) and the $x$ axis, determining the sign of $B_{66}$, is defined to be along an a direction.

The procedure is now straightforward using a computer. The molecular fields, (3), acting on the ith ion are evaluated assuming a certain structure of the moments (with the periods of seven or eight layers). The total effective single-ion Hamiltonian for the $i$ th ion (layer)

$$
\mathscr{H}^{i}=\mathscr{H}_{\mathrm{I}}^{i}+\mathscr{H}_{\mathrm{I}}^{i}-g \mu_{\mathrm{B}} \boldsymbol{H} . \boldsymbol{J}_{\boldsymbol{i}}
$$

(including the effect of an external field $\boldsymbol{H}$ ) is diagonalized determining the eigenenergies, $E_{v}^{i}$, and the corresponding eigenfunctions $\left|v^{i}\right\rangle$. The thermal expectation value of a operator, $\mathrm{O}_{l}^{m}$, is then given by

$$
\left\langle\mathrm{O}_{l}^{m}\right\rangle_{i}=\frac{1}{Z_{i}} \sum_{v=1}^{2 J+1}\left\langle v^{i}\left|\mathrm{O}_{l}^{m} \exp \left(-E_{v}^{i} / k_{\mathrm{B}} T\right)\right| v^{i}\right\rangle
$$

where

$$
Z_{i}=\sum_{v=1}^{2 J+1}\left\langle v^{i}\left|\exp \left(-E_{\mathrm{v}}^{i} / k_{\mathrm{B}} T\right)\right| v^{i}\right\rangle
$$

and the free energy of the $i$ th ion is

$$
F_{i}=-k_{\mathrm{B}} T \ln \left(Z_{i}\right)
$$

( $k_{\mathrm{B}}$ is Boltzmann's constant). This scheme is applied to every ion in the period. The (new) expectation values determined by (7) are introduced in the equations for the molecular fields, (3), and the calculations are repeated. The calculations are continued until a self-consistent solution is attained. Except close to the phase transitions convergence was obtained quite rapidly (during four to five cycles).

\section{Determination of the molecular-field parameters}

The five parameters which determine the MF Hamiltonian are the four crystal-field parameters and one exchange coupling constant. $B_{20}$ and $\mathscr{F}(\boldsymbol{Q})$ entirely dominate the stipulation of the Néel temperatures, $T_{\mathrm{N}}$ and $T_{\mathrm{N}_{2}}$. To the first order in $\left\langle J_{z}=J \mid \mathscr{H}_{1}^{i} J_{z}=J\right\rangle / k_{\mathrm{B}} T_{\mathrm{N}}$, the Néel temperature, $T_{\mathrm{N}_{\|}}$, for a sinusoidal ordering or the $c$ axis moment with a wavevector $Q$ is given by

$$
k_{\mathrm{B}} T_{\mathrm{N}}=\frac{1}{3} J(J+1) \mathscr{H}(Q)\left[1-\frac{4}{5}\left(J+\frac{3}{2}\right) J_{1} B_{20} / k_{\mathrm{B}} T_{\mathrm{N}}\right]
$$


(see for example Nagamiya 1967). Only the crystal-field parameter $B_{20}$ appears to first order of $1 / k_{\mathrm{B}} T$. This also holds true for the Néel temperature of the basal-plane component, $T_{\mathrm{N}_{\mathrm{H}}}$, however, the presence of a substantial first-order harmonic of the $c$ axis component ( $\equiv J \sigma_{1}^{z}$ ) at the transition implies a modification (Miwa and Yosida 1961, Nagamiya 1967):

$k_{\mathrm{B}} T_{\mathrm{N}_{\perp}}=\frac{1}{3} J(J+1) \mathscr{J}(Q)\left\{1+\frac{2}{5}\left(J+\frac{3}{2}\right) J_{1} B_{20} / k_{\mathrm{B}} T_{\mathrm{N}_{\perp}}-\frac{1}{60}\left(J^{2}+J+\frac{1}{2}\right)\left[J \sigma_{1}^{z} \mathscr{J}(Q) / k_{\mathrm{B}} T_{\mathrm{N}}\right]^{2}\right\}$

The insertion of the experimental values in (8) and (9) $\left(T_{\mathrm{N}}=84.4 \mathrm{~K}, T_{\mathrm{N}_{\mathrm{i}}}=52.4 \mathrm{~K}\right.$, and $\sigma_{1}^{z}=0.88$, Habenschuss et al 1974) leads to one useful solution $\left(B_{20}\right.$ has to be negative) $f(Q)=0.307 \mathrm{meV}$ and $B_{20}=-1.65 \times 10^{-2} \mathrm{meV}$ which are very close to our final fit. Within the accuracy of the calculations equation (8) is fulfilled and the equation (9) is obeyed with a correction of the order of $3-4 \%$.

Having determined $\mathscr{F}(Q)$ (or $\mathscr{F}_{0}$ ) and $B_{20}$ the remaining three crystal-field parameters are fixed by the magnetization data at $4.2 \mathrm{~K}$ (Rhyne et al 1968, Bozorth et al 1972). The mean value of the $c$ axis moment

$$
\left\langle\overline{J_{z}}\right\rangle=\frac{1}{n} \sum_{i=1}^{n=8}\left\langle J_{i}^{2}\right\rangle
$$

as a function of a field applied along the $c$ direction offers two conditions, and the magnitude of the hexagonal anisotropy, $B_{66}$, is adjusted so as to obtain agreement with the observed difference between the mean values of the moment, $\left\langle\bar{J}_{\|}\right\rangle$, parallel to a field of about $100 \mathrm{kOe}$ applied along either an $a$ or a $b$ direction (see figure 2). The cone angle of Er determined at zero field and at $4.2 \mathrm{~K}$ varies somewhat

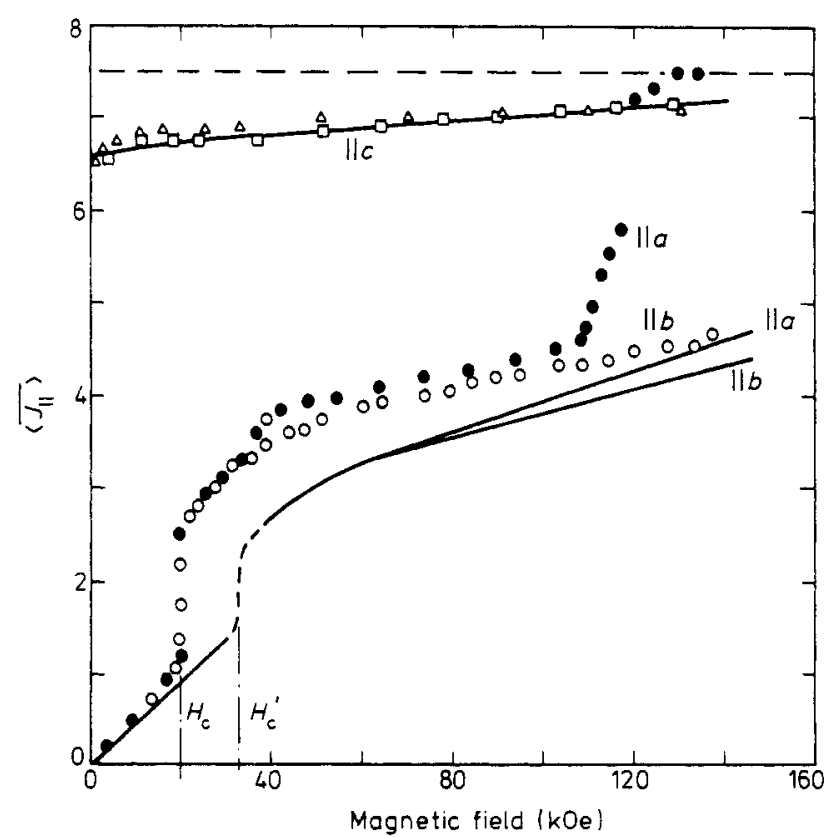

Figure 2. The mean value of the parallel components of the moments when a field is applied along an $a, b$, or $c$ direction in Er at $4.2 \mathrm{~K}$. The experimental results of Bozorth et al (1972) are shown, and the squares are the $c$ axis results of Rhyne et al (1968) scaled with a constant factor equal to 0.963 . The full curves display the MF results. 
Table 2. The effective crystal-field parameters derived by the MF calculations compared with the single-ion parameters of Er diluted in $\mathrm{Y}$ determined by $\mathrm{H} ø \mathrm{~g}$ and Touborg (1974) (in units of meV). If the fitting parameter $\left|B_{20}\right|$ is increased by $10 \%$ then the fit to the magnetization data at $4.2 \mathrm{~K}$ remains unaltered if $\left|B_{40}\right|$ is decreased by $18.4 \%$ and $B_{60}$ by $7.5^{\circ} \%$

\begin{tabular}{lcc}
\hline Parameter & Fit & $0.14^{\circ}{ }_{0}$ Er in Y \\
\hline$B_{20} \times 10^{2}$ & -1.75 & -2.66 \\
$B_{40} \times 10^{5}$ & -4.75 & 7.01 \\
$B_{60} \times 10^{6}$ & 1.06 & 2.09 \\
$B_{66} \times 10^{6}$ & -6.9 & -24.0 \\
\hline
\end{tabular}

from one experiment to the next (between $27 \cdot 7^{\circ}$ and $29 \cdot 6^{\circ}$ ). Here we use $\left\langle\overline{J_{z}}\right\rangle=6.590$ and the $c$ axis data of Rhyne et al (1968) shown in figure 2 have been scaled with a constant factor of 0.963 to agree with this choice. In figure 2 we have omitted the basal plane data of Rhyne et al as these results do not define properly an easy axis (but are otherwise in qualitative agreement with the results of Bozorth et al 1972).

The crystal-field parameters which are used in the MF calculations are given in table 2 . The intra-planar exchange constant $\mathscr{f}_{0}$ is included in table 1 (this value corresponds to $\mathscr{J}(\boldsymbol{Q})=0.304 \mathrm{meV}$ ). In table 2 the effective crystal-field parameters are compared with the single-ion parameters determined by Høg and Touborg (1974) from magnetization measurements on a single crystal alloy of Er diluted in $\mathrm{Y}$. The parameters determined by Høg and Touborg are not affected by two-ion couplings and may be considered as representative for the pure rare earth metal. The comparison in table 2 supports the tendency found in the case of $\mathrm{Tb}$ (Houmann et al 1975) that the two-ion correction to an effective crystal field parameter, $B_{l m}$, is important for all values of the rank $l$.

The paramagnetic Curie temperatures, $\theta_{1}=61.7 \mathrm{~K}$ and $\theta_{\perp}=32.5 \mathrm{~K}$, as measured by Green et al (1961), are not in agreement with the present set of parameters. The difference between $\theta$ and $\theta$. suggests a $B_{20}$ which is almost twice the value deduced from $T_{N}$ and $T_{N}$ The paramagnetic Curie temperatures are not determined with the same precision as the Néel temperatures. However, the possible discrepancy indicates the additional two-ion anisotropy

$$
-\frac{1}{2} \sum_{i \neq j} K(i j) J_{i}^{z} J_{j}^{z}
$$

to be present in Er. This axial two-ion coupling affects $T_{\mathrm{N}}$ and $\theta(\mathscr{H}(\boldsymbol{q})$ is replaced by $\mathscr{f}(q)+K(q)$ ) but has a very small influence on the spin wave energies of the cone phase (see Jensen 1974). The possibility of a two-ion coupling like $K(i j)$ being important introduces some uncertainties in the calculations as we cannot anticipate an 'effective' $B_{20}$ to account for all the effects of $K(i j)$ in the $c$ axis modulated phases. In spite of the discrepancy introduced by the paramagnetic Curie temperatures they may be utilized in an estimate of $\mathscr{I}(Q)-\mathscr{H}(0)$. Namely, $B_{20}=-(2.5 \pm 0.8) \times 10^{-2} \mathrm{meV}, \theta=61.7 \pm 5 \mathrm{~K}$, and $T_{\mathrm{N}}=84.4 \mathrm{~K}$ implies that $\mathscr{f}(Q)-\mathscr{f}(\mathrm{O})=0.10 \pm 0.03 \mathrm{meV}$ in agreement with $\mathscr{f}(q)$ shown in figure 1.

The MF ground state at $4 \cdot 2 \mathrm{~K}$. which is obtained using the parameters given in table 1 and 2 , deviates slightly from an ideal spin wave state. The moment $|\langle\boldsymbol{J}\rangle|$ is calculated to be a factor $C_{1}=0.991$ times $J$. This small reduction of the moment 
Table 3. The ratios $C_{1}$, defined in the text, calculated at $4.2 \mathrm{~K}$.

\begin{tabular}{llll}
\hline$C_{1}$ & $C_{2}$ & $C_{4}$ & $C_{6}$ \\
0.991 & 0.965 & 0.438 & 2.157 \\
\hline
\end{tabular}

from its maximum value corresponds to a similar reduction deduced in the spin wave systems Tb and Dy at zero temperature (see for example Jensen 1975). Defining

$$
\begin{aligned}
& C_{l}=\left\langle\mathrm{O}_{l}^{0}\right\rangle /\left[k_{l} J J_{l-1} P_{l}^{0}(\cos \theta)\right] \\
& k_{1}=1 \quad k_{2}=2 \quad k_{4}=8 \quad k_{6}=16
\end{aligned}
$$

where $P_{l}^{0}(\cos \theta)$ is the Legendre polynomials and $\theta$ the angle the moment makes with the $c$ axis, then $C_{l}$ should be equal to one in the case of a perfect spin wave system. A Hartree-Fock (HF) calculation in the cases of $\mathrm{Tb}$ and Dy (Jensen 1975) shows $\left|C_{l}-1\right|$ to increase strongly with $l$. This also holds true in the present MF calculation on $\mathrm{Er}$ as is evident from the numbers given in table 3. Although the MF approximation is not as well justified as the HF approximation (at low temperatures) the MF values of the ratios $C_{l}$ should be of the right order of magnitude.

In the analysis of the spin wave data in $\operatorname{Er}$ at $4.5 \mathrm{~K}$ (Jensen 1974) a $q$ independent anisotropy $L=20.0 \pm 6.0 \mathrm{meV}$ was deduced. In the case where $C_{l}$ differ from 1 the $\mathrm{HF}$ expression for $L$ is (Jensen 1974, 1975).

$$
\begin{aligned}
L=\left(1 / C_{1}\right)\left[6 C_{2} J_{1} B_{20}+60 C_{4} J_{3} B_{40}\left(7 \cos ^{2} \theta-1\right)+210 C_{6} J_{5} B_{60}\left(33 \cos ^{4} \theta\right.\right. \\
\left.\left.-18 \cos ^{2} \theta+1\right)+C_{1}^{2} J\{\mathscr{H}(Q)-\mathscr{I}(0)\}-C_{2}^{2} J \mathscr{K}_{22}(2 Q) \frac{1}{2}\left(3 \cos ^{2} \theta-1\right)\right] .
\end{aligned}
$$

Using the MF values of $C_{l}$ given in table 3 we obtain $L=23.7 \mathrm{meV}$. In (12) the contribution arising from $B_{60}$ entirely dominates. $L$ may be estimated also from the (experimental) slope of $\left\langle\bar{J}_{z}\right\rangle$ versus a field along the $c$ direction (figure 2). However, this estimate presupposes that $\mathrm{d} C_{l} / \mathrm{d} H_{z}$ is zero (Jensen 1975) in which case

$$
L=g \mu_{\mathrm{B}}\left(|\langle\boldsymbol{J}\rangle| \frac{\mathrm{d}\left\langle J_{z}\right\rangle}{\mathrm{d} H_{z}}\right)_{H_{z} \rightarrow 0}
$$

equal to approximately $15 \mathrm{meV}$ (see figure 2). When the moments are not along a symmetry direction $\left(\theta \neq 0\right.$ or $(\pi / 2)$ then $\mathrm{d} C_{l} / \mathrm{d} H_{z}$ may be nonzero and give rise to an important correction to (13). The $L$ value determined by equation (12) should be somewhat more reliable than the value deduced from the slope of $\left\langle\overline{J_{z}}\right\rangle$, and we may evidently conclude that the anisotropy constant $L$ deduced from the spin wave data is consistent with the present MF parameters.

\section{The magnetic structure of Er and comparison with experiments}

The $c$ axis moment of the $i$ th ion in the two high temperature phases may be written as follows

$$
\left\langle J_{i}^{2}\right\rangle=\sum_{p=1,3,5.7} A_{p} \sin \left(\mathrm{i} p \alpha_{0}+\beta_{p}\right)
$$

where $\alpha_{0}=(2 \pi / 7)$ considering a periodic structure with seven hexagonal layers. 
Neglecting the possibility of even harmonics we have

$$
\left.A_{p} \underset{\cos }{\operatorname{in}}\left(\beta_{p}\right)=\frac{2}{7} \sum_{i=1}^{7} \cos _{\sin }^{\cos \left(i p x_{0}\right.}\right)\left\langle J_{i}^{z}\right\rangle \quad p=1,3,5
$$

and the seventh harmonic appears as a ferromagnetic component $\left(A_{7}=\left\langle\bar{J}_{z}\right\rangle\right)$.

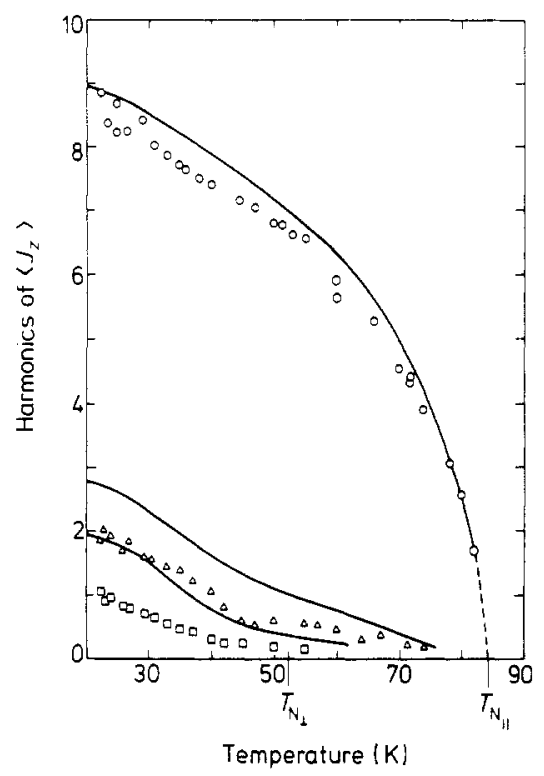

Figure 3. The amplitudes of the harmonics of the $c$ axis moments in $\mathrm{Er}$ as functions of temperature. The experimental results are those obtained by Habenschuss et al (1974) which are in close agreement with the results of Atoji (1974). The observed harmonics of higher order than five are omitted (the amplitudes are small). The full curves are the amplitudes deduced from the model calculations. 0 , 1st harmonic; $\Delta$, 3rd harmonic and $\square$ th harmonic.

In figure 3 we show the calculated magnitudes of the first, third and fifth harmonics of the $c$ axis moments as functions of temperature. Neither the calculated nor the experimental values of Habenschuss et al (1974) show any anomalies at the ordering temperature of the basal plane components, $T_{\mathrm{N}}$. The amplitudes of the harmonics were found to be very insensitive to the crystal-field parameters (when fixing $T_{N}$ to be equal $84.4 \mathrm{~K}$ ) implying that $\mathscr{f}^{\prime}(\boldsymbol{q})$ is responsible for most features of the temperature dependence of the harmonics. The first harmonic was unaffected also by the choice of the phase $\beta_{1}$ in (14), but this was not the case for the higher harmonics which vary as much as $50 \%$ when changing $\beta_{1}$. The calculated curves shown in figure 3 correspond to $\left|\beta_{1}\right|$ equal to about $45^{\circ}$ which led to values of the higher harmonics lying in between the two extremes. The seventh harmonic, which is omitted in figure 3, was calculated to have an amplitude smaller than 1 down to the lowest temperature (slightly larger than the experimental value). Although the calculated amplitudes of the harmonics are somewhat larger than the observed values of Habenschuss et al (1974) the qualitative behaviour is very similar.

Below $T_{N_{-}}$the basal-plane moments are nonzero, and the positional dependence of $\left\langle J_{\perp}\right\rangle$ is described in terms of harmonics equivalent to (14). A consideration of a structure for which the hodograph of the basal-plane moments is an ellipse (with 


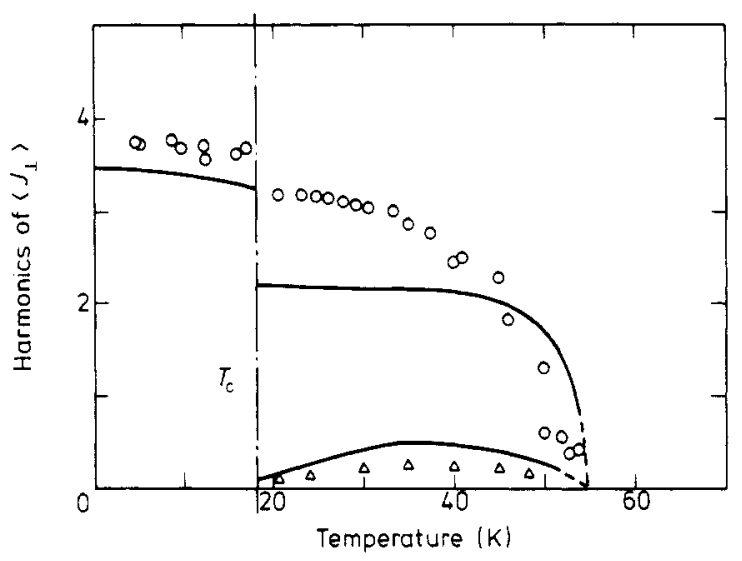

Figure 4. The amplitudes of the harmonics of the basal-plane moments in Er against temperature. The full curves are the MF results, and the experimental values are those of Habenschuss et al (1974). The fifth harmonic above $T_{c}$ is observed (and calculated) to have an amplitude somewhat smaller than that of the third harmonic. The results of Atoji (1974) are very similar except the basal-plane moments in the cone phase (below $T_{c}$ ) are slightly smaller (equal to the calculated moments) than the results of Habenschuss et al. 0.1 st harmonic and $\triangle 3$ rd harmonic.

the principal axes equal to $a_{1}$ and $a_{2}$ ) shows that if the direction of the major axis is equally distributed among all directions in the basal plane then the neutron diffraction pattern will be the same as if the hodograph had been a circle with the radius $\left[\left(a_{1}^{2}+a_{2}^{2}\right) / 2\right]^{1 / 2}$. The presence of a hexagonal anisotropy, $B_{66}$, may cause staggering effects (a preferred orientation of the ellipse) but as long as all $a$ directions are equivalent the diffraction pattern is unaltered. Consequently the amplitudes of the basalplane harmonics determined experimentally $\left\langle\left\langle J_{x}\right\rangle=\left\langle J_{y}\right\rangle\right)$ are compared, in figure 4 , with the geometric mean values of the calculated harmonics of $\left\langle J_{x}\right\rangle$ and $\left\langle J_{y}\right\rangle$. Whereas the calculated values of the higher harmonics are somewhat larger than the measured ones the first harmonic of $\left\langle J_{\perp}\right\rangle$ (below $40 \mathrm{~K}$ ) is deduced to have an amplitude which is smaller than the experimental value. These differences are presumably strongly correlated with the discrepancies present in the case of the $c$ axis moments.

Table 4. The calculated moments, $\left\langle J_{i}^{x}\right\rangle$, of the equilibrium configuration in $\mathrm{Er}$ at $40 \mathrm{~K}$. $i$ denotes one of the seven different hexagonal layers of the period. The relative lengths of the moments are given, and as an example is given the expansion of $\left\langle J_{i}^{z}\right\rangle$ as considered in the text.

\begin{tabular}{lcccl}
\hline$i$ & $\left\langle J_{i}^{x}\right\rangle$ & $\left\langle J_{i}^{y}\right\rangle$ & $\left\langle J_{i}^{z}\right\rangle$ & $\langle J J V J$ \\
\hline 1 & 4.129 & 0.050 & 2.461 & 0.641 \\
2 & 1.300 & 0.058 & 6.441 & 0.876 \\
3 & -0.638 & 0.057 & 6.654 & 0.891 \\
4 & -2.162 & 0.041 & 5.030 & 0.756 \\
5 & -2.201 & -0.046 & -5.571 & 0.799 \\
6 & -0.342 & -0.061 & -6.733 & 0.899 \\
7 & 1.729 & -0.049 & -6.090 & 0.844 \\
\hline$\left\langle J_{i}^{z}\right\rangle=7.895 \sin \left(i \times 51.43^{\circ}-44.8^{\circ}\right)+1.643 \sin \left(3 i \times 51.43^{\circ}-131.6^{\circ}\right)$ \\
$+0.809 \sin \left(5 i \times 51.43^{\circ}+151.3^{\circ}\right)+0.313 \sin \left(7 i \times 51.43^{\circ}+90^{\circ}\right)$
\end{tabular}




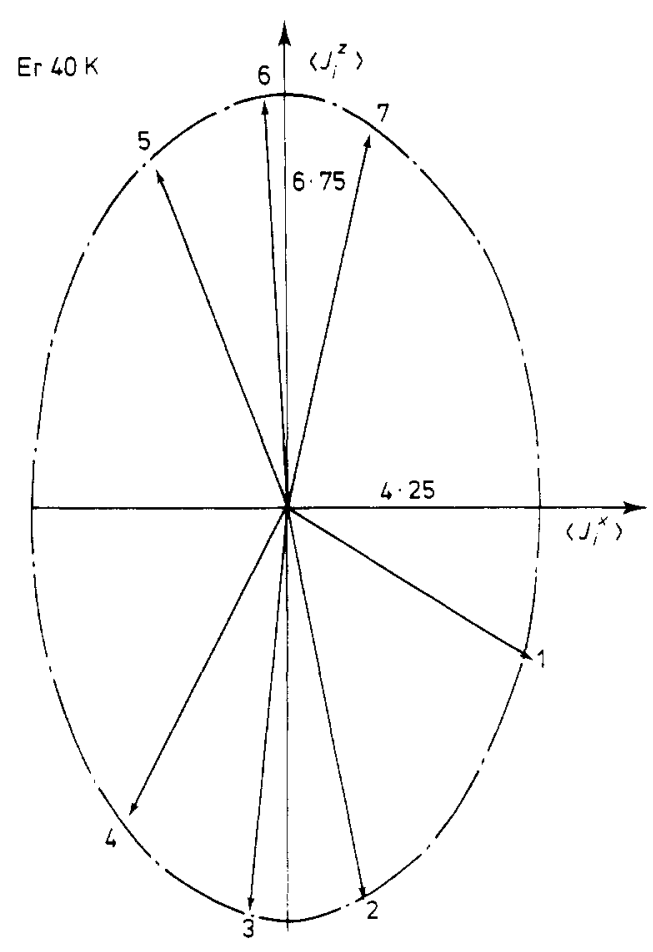

Figure 5. The calculated moments of $\mathrm{Er}$ at $40 \mathrm{~K}$ projected on the $x-z$ plane and displaced to a common origin. This is the configuration given by table 4 from which it appears that the $y$ axis moments are negligible. $i$ equal to $1,2, \ldots, 7$ designates the moment of the $i$ th hexagonal layer. The hodograph is very close to the ellipse that has its axes equal to 4.25 and 6.75 as displayed in the figure.

The all round agreement between the calculated and experimental diffraction pattern at $40 \mathrm{~K}$ indicates that the magnetic structure calculated at this temperature should be very similar to the real structure. In table 4 the calculated moments, $\left\langle J_{i}^{x}\right\rangle$, for the seven different planes are tabulated. As appearing from the table only one of the basal-plane components is of any importance implying that the hodograph of the total moments is very closely confined to the $x-z$ plane. In figure 5 is shown the $x-z$ plane of the hodograph at $40 \mathrm{~K}$, and it appears to be an almost perfect ellipse. Further, the calculations indicate that the staggering effect due to $B_{66}$ is very small.

The magnetic structure depicted in figure 5 was calculated to be the configuration present in the whole intermediate phase, namely, a hodograph of the moments which is an ellipse with its major axis close to the $c$ direction. No tendency towards tilting the ellipse by more than a few degrees was found. Between $18 \mathrm{~K}$ and $22 \mathrm{~K}$ the fundamental wavevector is observed to correspond precisely to an eight layer structure (Habenschuss et al 1974). This value of the wavevector favours a pure square-wave arrangement of the $c$ axis component (a constant length of the $c$ axis moments). Although the 'squaring up' is approached more closely by the calculated $c$ axis moments than observed experimentally we found that the length of the $c$ axis 
moments was still varying by $8 \%$ in the case of the eight layer structure. As far as the results for the cases of the seven and the eight layer structures were comparable no significant differences were detected.

The basal-plane configuration is clamped to the modulated structure of the $c$ axis moments. The basal-plane component attains its maximum value when the $c$ axis moment is smallest and vice versa in accordance with the plane elliptical arrangement shown in figure 5. This clamping effect immediately explains the observation of the relatively large intensities of the third and fifth harmonics in the diffraction pattern of the basal-plane moments. The structure shown in figure 5 implies a smaller variation of the length of the total moments than produced by the corresponding helical ordering of the basal-plane moments, and this holds true until the $c$ axis moments are very close to constitute a regular square wave.

Although the differences between the calculated and measured moments introduce some uncertainties these MF calculations strongly indicate that the 'squaring up' of the $c$ axis moments in $\mathrm{Er}$ above $T_{\mathrm{c}}$ is not approached sufficiently to justify a model of a helical ordering of the basal-plane moments. Instead it is much more likely that the elliptical arrangement of the moments in a plane perpendicular to the basalplane is an adequate model in the whole range of the intermediate phase.

The paramagnetic Curie temperatures suggest $\mathscr{I}(Q)-\mathscr{I}(0)$ to be equal to $0.10 \mathrm{meV}$ as in figure 1, however, a MF analysis of the conical phase indicates that this difference is smaller (as already noticed by Jensen 1974). The present set of parameters does not predict the ferromagnetic ordering of the $c$ axis moment below $T_{c}$. The free energy of the cone structure is calculated to be $-8.1 \mathrm{meV} /$ ion at zero temperature whereas the mean value of the free energy of the 'intermediate' phase is lower (about $-9.5 \mathrm{meV} /$ ion). The $\mathrm{MF}$ calculations predict a first-order transition at $T_{\mathrm{c}}$ only if $\mathscr{I}(Q)-\mathscr{I}(0)$ is reduced to about $0.06 \mathrm{meV}$. Using a value of this difference which is too large the intermediate phase becomes stable down to zero temperature, but other aspects of the behaviour of the metastable cone structure are not necessarily affected (as this discrepancy could be due to $K(q)$ ).

The effective hexagonal anisotropy, $B_{66}$, is relatively small and the calculated cone structure is only slightly distorted. If the effective $B_{66}$ had been of the same magnitude as the single-ion parameter determined by Høg and Touborg (1974, see table 2) then the fifth and seventh harmonics of the basal-plane moments induced by $B_{66}$ would have been detectable and distinct energy gaps due to $B_{66}$ would have been present in the spin wave spectrum. These effects are not observed which is consistent with the value of the effective $B_{\kappa \kappa}$ deduced from the magnetization data.

When a field is applied along the $c$ direction only the cone angle is changed. The application of a field in the basal plane implies a radical change of the structure. This situation has been analysed by Nagamiya and co-workers (Kitano and Nagamiya 1964, Nagamiya 1967). At small fields the spiraling of the basal-plane moments is only distorted slightly so as to produce a small ferromagnetic component along the field. At a critical field, $H_{\mathrm{c}}$, the distorted spiral changes abruptly into a fan structure in which arrangement the parallel component is close to a ferromagnetic alignment. This transition appears as the jump in the magnetization curve shown in figure 2 , at $H=H_{\mathrm{c}}=20 \mathrm{kOe}$. As the field is increased the amplitude of the oscillating basalplane component perpendicular to the field (the opening angle of the fan) decreases monotonically. According to the MF calculations of Nagamiya and co-workers a second-order transition to a pure ferromagnetic state with the moments lying at an 
angle near that of the original cone occurs at the field $H_{\mathrm{f}}$

$$
H_{\mathrm{f}}=\frac{\sin \theta}{g \mu_{\mathrm{B}}}\left\{J[\mathscr{I}(Q)-\mathscr{I}(0)]+\frac{1}{2} J\left[\mathscr{K}_{22}(2 Q)-\mathscr{K}_{22}(0)\right] \sin ^{2} \theta\right\}
$$

when including the effect of $\mathscr{K}_{22}(q)$ and neglecting $B_{66}$. The critical field, $H_{c}$, for the spiral to fan transition is calculated by Nagamiya and co-workers to be given approximately by

$$
H_{\mathrm{c}}=\frac{1}{2} H_{\mathrm{f}} .
$$

Using this equation we calculate $H_{\mathrm{c}}^{\prime}=33 \mathrm{kOe}$ with $\mathscr{J}(\boldsymbol{q})$ and $\mathscr{K}_{22}(q)$ of figure 1 . Direct model calculations agree with the value of $H_{\mathfrak{c}}^{\prime}$ by indicating the critical field to be around $35 \mathrm{kOe}$. The hexagonal anisotropy has a negligible effect on $H_{\mathfrak{c}}$, but the possible magnetoelastic couplings may contribute to the experimental value of $H_{\mathfrak{c}}$, however, these contributions are presumably also very small $(\approx 1 \mathrm{kOe})$. Thus the discrepancy between the experimental and calculated critical field has to originate from the two-ion couplings, and it suggests that the difference $\mathscr{y}(Q)-\mathscr{J}(0)$ has to be scaled with a factor of the order of $\frac{2}{3}$ as for the energy considerations above.

Except for the location of the critical field the calculated basal-plane magnetization data are in fair agreement with the experimental results (when displacing the calculated curves so that $H_{\mathrm{c}}$ and $H_{\mathrm{c}}^{\prime}$ coincide). In the model calculations the field, $H_{\mathrm{f}}$, for a complete ferromagnetic alignment was found to be about $100 \mathrm{kOe}$ which is somewhat larger than $H_{\mathrm{f}}=2 \mathrm{H}_{\mathrm{c}}^{\prime}=66 \mathrm{kOe}$ suggested by equation (15). This disagreement is presumably produced by the field dependence of $C_{l},(11)$ (Er is not a perfect spin wave system). The effect agrees qualitatively with the neutron experiment of Rhyne and Pickart (1971) who observed the fan structure to exist still in a field of $60 \mathrm{kOe}$ applied in the basal plane (which is more than $2 H_{\mathrm{c}}(\exp )=40 \mathrm{kOe}$ ). The observation of an abrupt change of the magnetization along the $a$ direction (easy axis) at a field of the order of $120 \mathrm{kOe}$ was not reproduced by the present calculations (magnetoelastic effects may play an important role in this transition), instead they predict the ferromagnetic alignment of the $c$ axis component to be unstable above $150 \mathrm{kOe}$ with respect to the formation of an oscillating moment.

\section{Discussion}

The magnetic moments in $\mathrm{Er}$ arrange themselves in different very complex configurations depending on the temperature. In spite of the complexity of the magnetic structure we have shown that it is possible to attack this ground state problem and obtain meaningful results using a simplifying MF approximation. A reasonable fit to most of the features of the neutron diffraction results and to the magnetization data (at $4.2 \mathrm{~K}$ ) was achieved using only five independent parameters. The explicit model calculation presented supports the earlier MF calculations by Miwa and Yosida (1961) and Nagamiya (1967) introducing only a new aspect to the transition at low temperatures between the fan and the ferromagnet occuring when a field is applied in the basal plane.

The existing discrepancies indicate that the MF Hamiltonian deduced is incomplete. However. the way in which improvements should be performed is not obvious. The comparison between the effective and the 'single-ion' crystal-field parameters in table 2 
and also the paramagnetic Curie temperatures indicate the presence of axial two-ion anisotropy as for example $K(\boldsymbol{q})$ defined by (10). The temperature dependence of the fundamental wavevector, $Q$ (Cable et al 1965, Habenschuss et al 1974, Atoji 1974) demonstrates that the two-ion coupling is temperature dependent (and/or that higherorder axial two-ion anisotropy is present). The high temperature estimate of $\mathscr{I}(Q)=\mathscr{I}(0)$ (plus $K(Q)-K(0)$ ) to be $0.10 \mathrm{meV}$ together with the smaller value of $\sim 0.06 \mathrm{meV}$ determined by $T_{\mathrm{c}}$ shows that this difference cannot be expected to be independent of the temperature. The calculated value of the critical field $H_{\mathrm{c}}$ at $4.2 \mathrm{~K}$ is about $50 \%$ larger than the experimental value which also suggests a smaller difference $\mathscr{I}(Q)-\mathscr{I}(0)$ (but without any contributions from $K(q)$ ). Such a demand interferes with the interpretation of the spin wave data (which determine $\mathscr{J}(q)$ rather than $\mathscr{f}(\boldsymbol{q})+K(\boldsymbol{q}))$. The spin wave dispersion relation in the $c$ direction of $\mathrm{Er}$ at $4.5 \mathrm{~K}$ may be reproduced by the two-ion Hamiltonian defined by (1) by a set of coupling parameters which predicts a critical field $H_{\mathrm{c}},(15)$, in agreement with experiments. This fit requires an anisotropy constant $L$, (12), equal to $30 \mathrm{meV}$ (whereas the magnetization data and these MF calculations agree with the value of $20 \mathrm{meV}$ deduced by Jensen 1974), and it does not produce the observed behaviour of the intensities of the inelastic scattered neutrons. In the analysis of the spin wave data (Jensen 1974) it was concluded that $\mathscr{K}_{22}(q)$ is an important factor (because of its weight); the presence of other kinds of anisotropic two-ion couplings was not excluded. The difference between the calculated and experimental values of $H_{\mathrm{c}}$ may be explained by introducing other types of two-ion anisotropy as well as by the change of $L$. Here we may add that the present MF calculations support the indications of a very complex nature of the two-ion coupling in Er.

In a discussion of the origin of the two-ion anisotropy in the rare earth metals (see for an example Birgeneau et al 1974) it is of vital interest to attempt a distinction between effects arising from tensor operator couplings for which the ranks of the tensors are both even (electric multipole couplings) or both odd (dominated by the exchange coupling). At present, such a differentiation is not possible in $\operatorname{Er} . \mathscr{K}_{22}(q)$ appears in the two-ion Hamiltonian, (1), as a pure quadrupole interaction, however, the spin wave energies of the cone phase are changed negligibly (less than $0.1 \mathrm{meV}$, and $H_{\mathrm{c}}^{\prime}$ is unaffected) if this anisotropy term is replaced by the exchange term:

$$
\begin{aligned}
&-\frac{1}{2} \sum_{i \neq j} \mathscr{K}_{22}(i j) {\left[16 J_{2}^{2}\left(3 \cos ^{2} \theta-1\right)\right]^{-1}\left\{[ J _ { z } ( J ^ { + } ) ^ { 2 } + ( J ^ { + } ) ^ { 2 } J _ { z } ] _ { i } \left[J_{z}\left(J^{-}\right)^{2}\right.\right.} \\
&\left.\left.+\left(J^{-}\right)^{2} J_{z}\right]_{j}+\mathrm{hc}\right\}
\end{aligned}
$$

$\left(\mathscr{K}_{22}(i j)\right.$ is kept unchanged). This replacement will not affect the present calculations very much either, because the contribution of $\mathscr{K}_{22}(i j)$ to the free energy (below $T_{N_{\perp}}$ ) is at maximum a factor of five smaller than that of the isotropic two-ion coupling.

The crystal-field parameters derived by the MF analysis of Er are effective, modeldependent parameters. Although these parameters are determined unambiguously by the magnetization data at $4.2 \mathrm{~K}$ and the two Néel temperatures the apparent necessity of introducing other kinds of two-ion anisotropy besides $\mathscr{K}_{22}(q)$ makes this determination arbitrary. Further, it is not possible to isolate the crystal-field parameters from intra-planar two-ion contributions (as for example the contribution $\mathscr{K}_{0}$ to $B_{40}$ ) because of the ferromagnetic ordering of each particular hexagonal layer. Considering these uncertainties the effective crystal-field parameters are in reasonable agreement with the single-ion parameters determined by Høg and Touborg (1974). 
The lack of detailed agreement between the calculated and the experimental neutron diffraction pattern just above $T_{\mathrm{c}}$ makes the discussion of the magnetic configuration present at these temperatures less conclusive. However, according to the trends of the MF calculations the magnetic arrangement between $18 \mathrm{~K}$ and about $30 \mathrm{~K}$ should be near a cycloid structure for which the hodograph is a circle perpendicular to the basal plane. Because of the presence of the higher harmonics this structure does not imply that the first harmonics of $\sqrt{2}\left\langle J_{\perp}\right\rangle$ and $\left\langle J_{z}\right\rangle$ are of equal magnitude (for instance the minor axis in figure 5 is almost twice the value suggested by the first harmonic of $\left.\left\langle J_{i}\right\rangle\right)$. This configuration is equivalent to the tilted helix considered by Sherrington (1973) with the tilt angle equal to $90^{\circ}$. The elementary excitations in this temperature range should be mostly spin-wave like as in the model considered by Sherrington, although longitudinal spin excitations might also be important. However, the complexity of the ground state configuration will make it very difficult to extract any useful information from the inelastic neutron scattering. At higher temperatures the length of the magnetic moments is varying and the tilt-helix model is no longer adequate.

The strong tendency of the moments in the intermediate phase to be confined within a plane, as indicated by the MF calculations, is not easily detected experimentally. The application of an external field will produce an effect that is similar to the case in which the basal-plane moments are spiraling in a regular fashion. Magnetic dipolar forces may be able to create a preferred orientation of the planes, when using a sample which is not cylindrical with respect to the $c$ axis, however, this effect is presumably quite small.

\section{Acknowledgments}

I am very much indebted to $O$ Jepsen for his assistance with the computer programme. Helpful discussions with P Bak, A P Young and R J Elliott are gratefully acknowledged.

\section{References}

Atoji M 1974 Solid St. Commun. 14 1047-50

Birgeneau R J. Kjems J K. Shirane G and Van Uitert L G 1974 Phys. Rev. B $102512-34$

Bozorth R M, Clark A E and Van Vleck J H 1972 Int. J. Magn. 2 19-31

Cable J W, Wollan E O, Koehler W C and Wilkinson M K 1965 Phys. Rev. 140 A 1896-902

Green R W, Legvold S and Spedding F H 1961 Phys. Rev. 122 827-30

Habenschuss M, Stassis C. Sinha S K, Deckman H W and Spedding F H 1974 Phys. Rev, B 10 1020-6 Houmann J G, Jensen J and Touborg P 1975 Phys. Rev. B 12 332-44

Høg J and Touborg P 1974 Phys. Rel: B $92920-8$

Jensen J 1974 J. Phys. F: Metal Phys. 4 1065-72

1975 J. Phys. C. Solid St. Phys. 8 2769-80

Kitano $Y$ and Nagamiya T 1964 Prog. Theor. Phys. 31 1-43

Lindgărd P A and Danielsen O 1974 J. Phys. C: Solid St. Phys. 7 1523-35

Miwa H and Yosida K 1961 Prog. Theor. Phys. 26 693-721

Nagamiya T 1967 Solid State Physics ed F Seitz and D Turnbull (New York: Academic Press) vol 20 pp 305-411

Nicklow R M, Wakabayashi N, Wilkinson M K and Reed R E 1971 Phys. Rev. Lett. 27 334-7

Rhyne J J, Foner S, McNiff E J and Doclo R 1968 J. Appl. Phys. 39 892-3

Rhyne J J and Pickart S J 1971 Magnetism and Magnetic Materials ed C D Graham Jr and J J Rhyne (AIP Conf. Proc. No. 5) pp 1436-40

Sherrington D 1973 J. Phys. C: Solid St. Phys. $61037-53$ 